\title{
ON HANDLE DECOMPOSITIONS AND DIFFEOMORPHISMS
}

\author{
BY \\ MAX K. AGOSTON(1)
}

In this paper we are concerned with two questions which arise naturally in the study of manifolds. One has to do with whether certain types of handle decompositions of a manifold are unique; and the other, under what circumstances they are preserved by diffeomorphisms. The corollaries to Theorem 1 and 2 are partial answers to these questions. Corollary 2 may prove useful for the general study of diffeomorphisms. We shall begin with some notation.

$\boldsymbol{R}^{k}$ shall denote real $k$-dimensional vector space with unit disc $D^{k}$ and unit sphere $S^{k-1}$. Let $I=[0,1]$. All manifolds are assumed to be compact and $C^{\infty}$. Homology groups are taken with integer coefficients. $\approx$ means either diffeomorphic or isotopic. By a nice Morse function on a manifold $M^{n}$ (see [5, p. 44]) we shall mean a function $\eta: M^{n} \rightarrow \boldsymbol{R}$ which has only nondegenerate critical points, and if $p$ is a critical point, then $\eta(p)=$ index $p$. It is well known that in this case $M_{k}(\eta) \equiv \eta^{-1}[0, k+1 / 2]$ is a $n$-submanifold of $M$ for $0 \leqq k \leqq n$, and

$$
M_{k}(\eta)=M_{k-1}(\eta) \cup_{\phi_{1}}\left(D^{k} \times D^{n-k}\right) \cup \cdots \cup_{\phi_{p}}\left(D^{k} \times D^{n-k}\right),
$$

where $\phi_{j}: \partial D^{k} \times D^{n-k} \rightarrow \eta^{-1}[k-1 / 2]$ are imbeddings. Call $\eta$ minimal if the number of critical points of index $k$ is equal to $p(k)+q(k)+q(k-1)$, where $p(k)$ is the torsionfree rank of $H_{k} M$ and $q(k)$ is the rank of the torsion subgroup of $H_{k} M$ (see [6, §6]).

From now on $M^{n}$ will be a closed simply-connected manifold with $n \geqq 6$.

Theorem 1. Let $\eta_{j}$ be minimal nice Morse functions on $M, j=1,2$. Assume that $H_{i_{s}} M$ is torsion-free for $0=i_{1}<i_{2}<\cdots<i_{t-1}<i_{t}=n$. Then there is a diffeomorphism $H: M \rightarrow M$ satisfying

(i) $H \approx$ identity,

(ii) $H\left(M_{i_{s}}\left(\eta_{1}\right)\right)=M_{i_{s}}\left(\eta_{2}\right)$, for $1 \leqq s \leqq t$.

TheOREM 2. Let $F: M \rightarrow M$ be a diffeomorphism and $\eta_{1}$ any minimal nice Morse function on $M$. Assume that $F_{*} \mid$ torsion $\left(H_{i_{s}} M\right)=$ identity for $0=i_{1}<i_{2}<\cdots<i_{t-1}<i_{t}$ $=n$. Then $F$ is isotopic to a diffeomorphism $F^{\prime}$ such that $F^{\prime}\left(M_{i_{s}}\left(\eta_{1}\right)\right)=M_{i_{s}}\left(\eta_{1}\right)$, for $1 \leqq s \leqq t$.

REMARK. The existence of minimal nice Morse functions on $M$ is guaranteed by $[6, \S 6]$.

Received by the editors June 12, 1967.

(1) This research was partially supported by a National Science Foundation Fellowship. 
As an immediate consequence of Theorems 1 and 2 we get the following corollaries.

Corollary 1. Assume that $H_{*} M$ is torsion-free. Let $\eta_{j}$ be minimal nice Morse functions on $M, j=1$, 2. Then $M_{i}\left(\eta_{1}\right) \approx M_{i}\left(\eta_{2}\right)$, for $0 \leqq i \leqq n$ (i.e., minimal handle decompositions of $M$ are unique up to diffeomorphisms).

Corollary 2. Let $F: M \rightarrow M$ be a diffeomorphism such that $F_{*} \mid$ torsion $H_{*} M$ $=$ identity. Let $\eta$ be a minimal nice Morse function on $M$. Then $F \approx F^{\prime}$, where $F^{\prime}\left(M_{i}(\eta)\right)=M_{i}(\eta)$, for $0 \leqq i \leqq n$ (i.e., up to isotopy, most diffeomorphisms of $M$ are "level-preserving").

In order to prove Theorems 1 and 2 we begin with a lemma.

Lemma. Let $\eta_{1}$ and $\eta_{2}$ be minimal nice Morse functions on $M$. Define $M_{i}^{j} \equiv$ $\eta_{j}^{-1}[0, i+1 / 2]$ and suppose that $M_{k}^{1}=M_{k}^{2}=A$ for some fixed $k, 0 \leqq k<n$. Let $k<l<$ $n-2$.

(a) If $H_{l} M$ is torsion-free, then there is a diffeomorphism $H$ of $M$ such that

(i) $H \approx$ identity,

(ii) $H \mid A=$ identity,

(iii) $H\left(\dot{M}_{l}^{1}\right)=M_{l}^{2}$.

(b) Assume $\eta_{2}=\eta_{1} F^{-1}$, where $F$ is a diffeomorphism of $M$ with $F_{*} \mid$ torsion $\left(H_{l} M\right)$ $=$ identity. Then there is a diffeomorphism $H$ of $M$ satisfying (i)-(iii) in part (a).

Proof. (a) The following easy general position argument shows that there is a diffeomorphism $h_{1}$ of $M$ satisfying (i), (ii) and $h_{1}\left(M_{l}^{2}\right) \subset \operatorname{int}\left(M_{l}^{1}\right) \equiv$ interior of $M_{l}^{1}$ : If $M_{l}^{2} \subset$ int $M_{q}^{1}$, for $q>l$, write $M_{q}^{1}=M_{q-1}^{1} \cup_{\psi_{1}}\left(D^{q} \times D^{n-q}\right) \cup \cdots \cup_{\psi_{p}}\left(D^{q} \times D^{n-q}\right)$, with respect to imbeddings $\psi_{j}: \partial D^{q} \times D^{n-q} \rightarrow \partial M_{q-1}^{1}$. Now as a cell complex $M_{l}^{2}$ has dimension at most $l$, so that $q>l$ implies that we may deform $M_{l}^{2}$ so that $M_{l}^{2} \cap\left(0 \times D^{n-q}\right)_{j}=\varnothing$; but once we have this, it is easy to push $M_{l}^{2}$ into int $M_{q-1}^{1}$. Repeating this process for each $q>l$ we get $h_{1}$.

Let $B \equiv h_{1}\left(M_{l}^{2}\right)$ and let $f: B \rightarrow M$ be the inclusion map. Consider

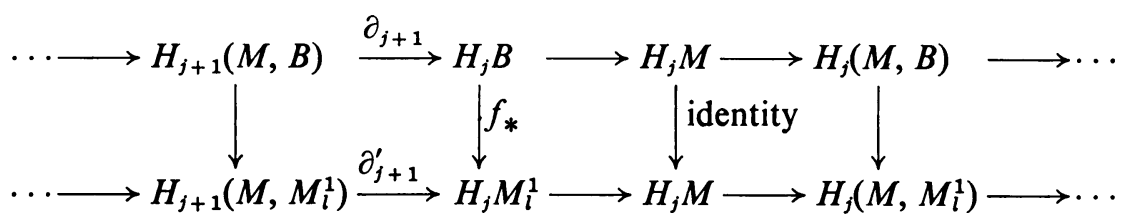

Since $H_{j}(M, B)=0=H_{j}\left(M, M_{l}^{1}\right)$ for $j \leqq l, f_{*}$ is an isomorphism for $j<l$. But $\partial_{l+1}=0$ $=\partial_{l+1}^{\prime}$, because $H_{l} M$ is torsion-free. Thus $f_{*}$ is an isomorphism for all $j$. By the Whitehead theorem (see [3, Theorem 10.1]), $f$ is a homotopy equivalence.

Claim. $\pi_{1} Y=\pi_{1} \partial M_{l}^{1}=\pi_{1} \partial B=0$, where $Y=$ closure of $M_{l}^{1}-B$.

Assuming this for the moment, we get by [5, Theorem 9.1] that $Y \approx \partial B \times I$. Thus, using the $I$-coordinate it should be clear how to define a diffeomorphism $h_{2}$ of $M$ satisfying (i), (ii) and $h_{2}(B)=M_{l}^{1}$. Let $H=h_{2} h_{1}$. Then $H$ is the desired map. 
To prove the claim, let $g: S^{1} \rightarrow Y$. Since $\pi_{1} M_{l}^{1}=0=\pi_{1} M$, there is a $\tilde{g}: D^{2} \rightarrow M_{l}^{1}$ with $\tilde{g} \mid S^{1}=g$. But $l+2<n$ and so we can push $\tilde{g}$ into $Y$ by a general position argument similar to the one at the beginning of this proof. Therefore, $g \simeq 0$ in $Y$, i.e., $\pi_{1} Y=0$. To see that $\pi_{1} \partial M_{l}^{1}=0$ we use induction $\left(\pi_{1} \partial B=0\right.$ by an analogous argument). Clearly, $\pi_{1} \partial M_{0}^{1}=\pi_{1} \partial M_{1}^{1}=\pi_{1} S^{n-1}=0$, because $n>2$ and $\pi_{1} M=0$. Now $\partial M_{j}^{1}$ is obtained from $\partial M_{j-1}^{1}$ by cutting out a certain number of disjoint $S^{j-1} \times D^{n-j}$ and adding an equal number of $D^{j} \times S^{n-j-1}$. If $\pi_{1} \partial M_{j-1}^{1}=0$, for $1<j<n$, then Van Kampen's theorem [2, Theorem 6.4.3] shows that $\pi_{1} \partial M_{j}^{1}=0$. This proves that $\pi_{1} \partial M_{j}^{1}=0$ for $0 \leqq j \leqq n$, and finishes the proof of part (a).

(b) The method of part (a) breaks down only at one point; namely, we do not know that $\partial_{l+1}=0=\partial_{l+1}^{\prime}$. Hence we must give a special argument to make $f$ into a homotopy equivalence. Specifically, assume that $F\left(M_{l}^{1}\right) \subset \operatorname{int} M_{l}^{1}$ and let $f \equiv F \mid M_{l}^{1}: M_{l}^{1} \rightarrow M_{l}^{1}$. Again, $f_{*}$ is an isomorphism for $j<l$. Consider

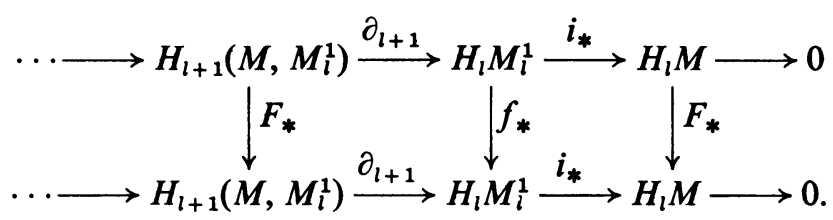

Write

$$
\begin{aligned}
H_{l} M_{l}^{1} & =\oplus \sum_{s=1}^{p(l)}\left\{x_{s}\right\} \oplus \sum_{s=1}^{q(l)}\left\{y_{s}\right\}, \\
H_{l} M & =\oplus \sum_{s=1}^{p(l)}\left\{x_{s}^{\prime}\right\} \oplus \sum_{s=1}^{q(l)}\left\{y_{s}^{\prime}\right\}, \quad k_{s} \equiv \text { order of } y_{s}^{\prime} .
\end{aligned}
$$

Note that $\operatorname{ker} f_{*} \subset \operatorname{ker} i_{*} \subset \sum_{s=1}^{q(l)}\left\{y_{s}\right\}$ and $f_{*}\left(\sum_{s=1}^{q(l)}\left\{y_{s}\right\}\right) \subset \sum_{s=1}^{q(l)}\left\{y_{s}\right\}$. Let $f_{*} y_{s}=$ $\sum_{j=1}^{q(l)} c_{s j} y_{j}$, and set $C \equiv\left(c_{s j}\right)$. We may assume without loss of generality that $i_{*} x_{s}=x_{s}^{\prime}$ and $i_{*} y_{s}=y_{s}^{\prime}$. Then our hypothesis shows that $y_{s}^{\prime}=i_{*} y_{s}=F_{*} i_{*} y_{s}=i_{*} f_{*} y_{s}=$ $i_{*} \sum_{j=1}^{q(l)} c_{s j} y_{j}=\sum_{j=1}^{q(l)} c_{s j} y_{j}^{\prime}$, i.e., $c_{s j} \equiv \delta_{s j}\left(k_{j}\right)$. Our task will be to modify $F$ so that $c_{s j}=\delta_{s j}$.

Put $c_{s j}=\delta_{s j}+a_{s j} k_{j}$, and let

$$
\begin{gathered}
M_{j}^{1}=M_{j-1}^{1} \cup_{\psi_{1, j}}\left(D^{j} \times D^{n-j}\right) \cup \cdots \cup_{\psi_{t j}, j}\left(D^{j} \times D^{n-j}\right), \\
H_{j}\left(M_{j}^{1}, M_{j-1}^{1}\right)=\oplus \sum_{s=1}^{t_{j}}\left\{z_{s}^{j}\right\},
\end{gathered}
$$

where $t_{j}=p(j)+q(j)+q(j-1)$ and $z_{s}^{j}$ corresponds to $\left(D^{j} \times 0\right)_{\psi_{s, j}}$. Consider

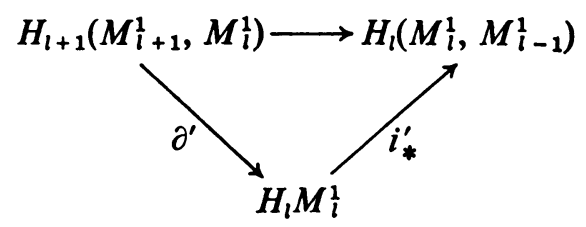

We may assume (see $[6, \S 6])$ that $i_{*}^{\prime} x_{s}=z_{s}^{l}, i_{*}^{\prime} y_{s}=z_{s+p(l)}^{l}$, and $\partial^{\prime} z_{s+p(l+1)+q(l+1)}^{l+1}=k_{s} y_{s}$. 
Let

$$
\begin{aligned}
& T^{s, t} \equiv F\left[\left(D^{l} \times 0\right)_{\psi_{s}+p(l), l}\right] \cap\left(0 \times D^{n-l}\right)_{\psi_{t+p(l), l},} \\
& T_{0}^{s, t} \equiv\left(\partial D^{l+1} \times 0\right)_{\psi_{s+p(l+1)+q(l+1), l+1}} \cap\left(0 \times \partial D^{n-l}\right)_{\psi_{t+p(l), l}} .
\end{aligned}
$$

By a general position argument we can make $T^{s, t}$ and $T_{0}^{s, t}$ consist of only a finite number of points. In fact, using methods of Whitney (e.g., see [1, §7]), we may suppose that $\left|T^{s, t}\right|=\left|c_{s t}\right|,\left|T_{0}^{s, s}\right|=k_{s}$, and $T_{0}^{s, t}=\varnothing$, for $s \neq t(|X|$ denotes the cardinality of $X$ ).

We shall now describe isotopies $h_{u}^{ \pm}$, with $h_{0}^{ \pm}=$identity $=h_{u}^{ \pm} \mid A$, so that if we let $F^{\prime}=h_{1}^{ \pm} F$ and define $C^{\prime}=\left(c_{s t}^{\prime}\right)$ similarly to $C$, then $c_{s_{1} t_{1}}^{\prime}=c_{s_{1} t_{1}}$, for $s_{1} \neq s, t_{1} \neq t$, and $c_{s t}^{\prime}=c_{s t} \pm k_{t}$. Using such isotopies it should be clear how to deform $F$ so that $c_{s t}=\delta_{s t}$.

The Construction of $h_{u}^{ \pm}$. Let $p \in T^{s, t}$ and $q \in T_{0}^{t, t}$. Let $\alpha: D^{n} \rightarrow M^{n}$ be an

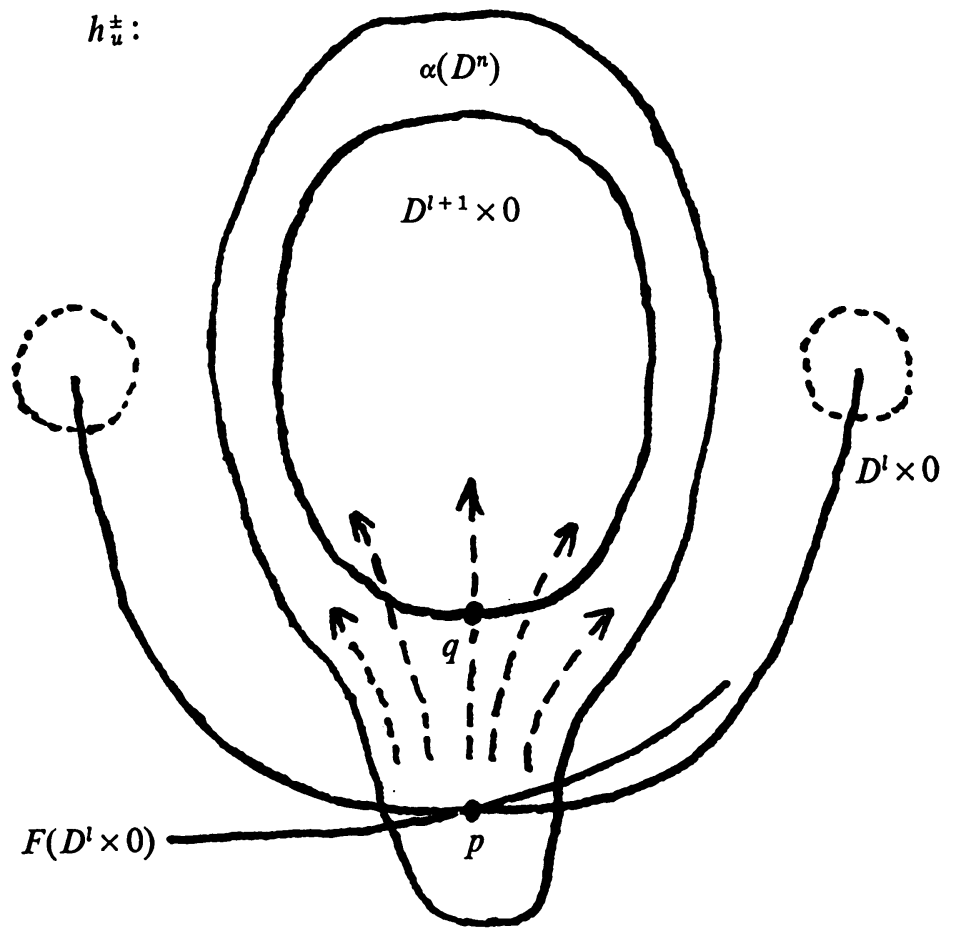

imbedding with the following properties:

(1) $\left(D^{l+1} \times 0\right)_{\psi_{t+p(l+1)+q(l+1), l+1}} \subset$ int $\alpha\left(D_{0}^{l+1}\right)$, where

$$
D_{0}^{l+1} \equiv\left\{\left(x_{1}, \ldots, x_{l+1}\right) \in D^{l+1} \mid x_{l+1} \geqq 0\right\},
$$

(2) $\alpha\left(D^{l+1}\right) \cap F\left[\left(D^{l} \times 0\right)_{\psi_{s}+p(l), l}\right]=\alpha\left(D^{l}\right)$,

(3) If $U \equiv F\left(M_{l}^{1}\right) \cap \alpha\left(D^{n}\right)$, then $U$ is a ball neighborhood of $p$ with $U \subset$ $\left(D^{l} \times D^{n-l}\right)_{\psi_{t}+p(l), l}$ and $U \cap T^{s, t}=\{p\}$, 
(4) $\alpha\left(D^{n}\right) \cap\left(D^{l} \times D^{n-l}\right)_{\psi_{j}+p(l), l}=\varnothing$, for $j \neq t$,

(5) $\alpha\left(\partial D^{l+1}\right) \subset$ int $M_{l}^{1}$.

$h_{u}^{+}$is then the isotopy which pushes $\alpha\left(D^{l}\right)$ to $\alpha\left(D_{+}^{l}\right)$, where $D_{+}^{l} \equiv D_{0}^{l+1} \cap S^{l} . h_{u}^{-}$is defined in essentially the same manner, except that one must give $\alpha\left(D^{l}\right)$ first a small twist in order to change the orientation.

Thus, assume now that $c_{s t}=\delta_{s t}$. By previous observations we get that $f_{*}$ is a monomorphism. A little diagram chasing shows that it is also onto, since it is already onto $\sum_{s=1}^{q(l)}\left\{y_{s}\right\}$. Therefore $f_{*}$ is an isomorphism and $f$ is a homotopy equivalence. The rest of the proof goes as in part (a). This finishes the lemma.

We are now ready to prove our theorems, keeping the notation of the lemma.

Proof of Theorem 1. By [4] we can find a diffeomorphism $H_{i_{1}}$ of $M$ isotopic to the identity and $H_{i_{1}}\left(M_{0}^{1}\right)=M_{0}^{2}$. Suppose inductively that we have defined a sequence of diffeomorphisms, $\left\{H_{i_{s}} \mid s=1,2, \ldots, k\right\}$, of $M$ satisfying

(1) $H_{i_{s}} \approx H_{i_{s-1}}$,

(2) $H_{i_{s}}\left|M_{i_{s-1}}^{1}=H_{i_{s-1}}\right| M_{i_{s-1}}^{1}$,

(3) $H_{i_{s}}\left(M_{i_{s}}^{1}\right)=M_{i_{s}}^{2}$.

Let $\eta_{3}=\eta_{1} H_{i_{k}}^{-1}$ and $M_{i}^{3} \equiv \eta_{3}^{-1}[0, i+1 / 2]$. Then $M_{i_{k}}^{3}=M_{i_{k}}^{2}$. Assume that $1 \leqq i_{k}$ $<i_{k+1}<n-2$. Applying part (a) of the lemma to $\eta_{3}$ and $\eta_{2}$ we obtain a diffeomorphism $h$ of $M$ with $h \approx$ identity, $h \mid M_{i_{k}}^{2}=$ identity, and $h\left(M_{i_{k+1}}^{3}\right)=M_{i_{k+1}}^{2}$. Define $H_{i_{k+1}} \equiv h H_{i_{k}}$. It is easily checked that $H_{i_{k+1}}$ satisfies (1)-(3).

If $n-2 \leqq i_{k+1} \leqq n$, let $\eta_{j}^{*} \equiv n-\eta_{\text {, and }} M_{i}^{j *} \equiv \eta_{j}^{*-1}[0, i+1 / 2]$. (Observe that $\eta_{j}^{*}$ is also a minimal nice Morse function.) Using [4] we can find a diffeomorphism $h_{1}$ of $M$ such that $h_{1} \approx$ identity, $h_{1} \mid M_{i_{k}}^{2}=$ identity, and $h_{1}\left(M_{0}^{3 *}\right)=M_{0}^{2 *}$. If $i_{k+1}=n-1$, define $H_{i_{k+1}}=h_{1} H_{i_{k}}$. If $i_{k+1}=n-2$, apply part (a) of the lemma to $\eta_{3}^{*} h_{1}^{-1}$ and $\eta_{2}^{*}$ with $l=1$ to get a diffeomorphism $h_{2}$ of $M$ with the property that $h_{2} \approx$ identity, $h_{2} \mid M_{0}^{2 *}=$ identity, and $h_{2}\left(h_{1}\left(M_{1}^{3 *}\right)\right)=M_{1}^{2 *}$. Furthermore, the proof of the lemma shows that we may suppose that $h_{2} \mid M_{i_{k}}^{2}=$ identity. Define $H_{i_{k+1}}=h_{2} h_{1} H_{i_{k}}$. Finally, if $i_{k+1}=n$, define $H_{i_{k+1}}=H_{i_{k}}$. In all cases, $H_{i_{k+1}}$ satisfies (1)-(3). This finishes our inductive definition of the $H_{i_{s}}$ and $H \equiv H_{n}$ satisfies the conclusion of the theorem.

Proof of Theorem 2. Let $\eta_{2} \equiv \eta_{1} F^{-1}$ and apply part (b) of the lemma in the proof of Theorem 1 instead of part (a) to obtain a diffeomorphism $H: M \rightarrow M$ such that $H \approx$ identity and $H\left(M_{i_{s}}^{1}\right)=M_{i_{s}}^{2}$. Let $F^{\prime}=H^{-1} F$.

At first glance, one might be led to conjecture that the condition, $F_{*} \mid$ torsion $H_{l} M$ $=$ identity, in the lemma is unnecessary; however, the following example shows that it is not:

Let $M^{8} \equiv$ double of $\left(S^{2} \times D^{6} \cup_{\alpha}\left(D^{3} \times D^{5}\right)\right)$, where $\alpha: S^{2} \times D^{5} \rightarrow S^{2} \times S^{5}$ is an imbedding with $\left[\alpha\left(S^{2} \times 0\right)\right]=5\left[S^{2} \times 0\right] \in H_{2}\left(S^{2} \times D^{6}\right)$. Let $\eta: M \rightarrow R$ be a minimal nice Morse function such that $M_{2}=S^{2} \times D^{6}$ and $M_{3}=S^{2} \times D^{6} \cup_{\alpha}\left(D^{3} \times D^{5}\right)$, where $M_{i} \equiv M_{i}(\eta)$. Construct an imbedding $h: M_{2} \rightarrow M_{2}$ such that $\left[h_{2}\left(S^{2} \times 0\right)\right]$ $=3\left[S^{2} \times 0\right] \in H_{2} M_{2}$. One can extend $h$ to an imbedding $M_{2} \cup_{\alpha}\left(D^{3} \times 0\right) \rightarrow M_{3}$. But $\pi_{2} \mathrm{SO}_{5}=0$, and so we can thicken the handle to get an imbedding $h: M_{3} \rightarrow M_{3}$. 
Now $H_{2} M_{3} \approx H_{2} M \approx(a)$, where $a$ has order 5 , and $h_{*}(a)=3 a$ by construction. Therefore $h$ is a homotopy equivalence and we may assume $h$ is a diffeomorphism of $M_{3}$. Let $F: M \rightarrow M$ be the double of $h$. Then $F_{*} \mid H_{2} M \neq$ identity. However, if $F \approx F^{\prime}$ and $F^{\prime}\left(M_{2}\right)=M_{2}$, then we must have $F_{*}\left|H_{2} M=F_{*}^{\prime}\right| H_{2} M= \pm$ identity because $\mathrm{H}_{2} \mathrm{M}_{2} \approx Z$. Thus $(M, F)$ is the example that we wanted. In fact, this also shows that Theorem 1 is the best possible (set $\eta_{1}=\eta$ and $\eta_{2}=\eta F$ ).

Finally, although we just saw that Theorem 1 is the best possible, this still leaves open the interesting question whether, despite this, the manifolds $M_{i}\left(\eta_{1}\right)$ and $M_{i}\left(\eta_{2}\right)$ are diffeomorphic anyway.

\section{REFERENCES}

1. G. De Rham, Torsion et type simple d'homotopie, Séminaire G. de Rham, 1963-1964, Faculté des Sciénces de l'Université de Lausanne.

2. P. J. Hilton and S. Wylie, Homology theory, Cambridge Univ. Press, New York, 1960.

3. Sze-Tsen Hu, Homotopy theory, Academic Press, New York, 1959.

4. J. Milnor, Differentiable structures, Mimeographed notes, Princeton Univ., Princeton, N. J., 1961.

5. - Lectures on the h-cobordism theorem, Princeton Univ. Press, Princeton, N. J., 1965.

6. S. Smale, On the structure of manifolds, Amer. J. Math. 84 (1962), 387-399.

YALE UNIVERSITY,

New Haven, Connecticut

WESLEYAN UNIVERSITY, Middletown, CONNECTiCUT 